\title{
Three Inverted Meckel's Diverticula Cases with Ileus Diagnosed Preoperatively and Treated Laparoscopically
}

\author{
Kazuya Naritomi*, Yuya Tanaka, Hiroki Ureshino, Yoshihiro Uchino, Motohisa Kuwahara, \\ Yuhei Kitazato, Masahiro Kawabata, Masae Mano \\ Department of Surgery, Saiseikai Futsukaichi Hospital, Fukuoka, Japan \\ Email: ${ }^{*}$ k-naritomi@saiseikai-futsukaichi.org
}

Received 22 December 2015; accepted 13 February 2016; published 16 February 2016

Copyright (C) 2016 by authors and Scientific Research Publishing Inc.

This work is licensed under the Creative Commons Attribution International License (CC BY). http://creativecommons.org/licenses/by/4.0/

(c) (i) Open Access

\begin{abstract}
We have experienced three inverted Meckel's diverticula with ileus that are diagnosed before surgery and treated with laparoscopic surgery. The patients were 22, 26, and 29 years of age. The history of ileus for each patient differed, being the first experience for one patient, the second for another, and the third experience for the remaining patient. None of these patients had a history of abdominal surgery. Conservative therapy eventually improved the existing symptoms, but the cause of past ileus remained unclear. Elective surgery was performed 12 days from the onset of symptoms on two of the patients after improving the ileus by inserting a long tube into the bowel. Emergency surgery was performed on the same day of admission on the remaining case that involved moderate expansion of intestine. In these patients, the first trocar was inserted into the umbilicus region. Laparoscopic surgery was then performed with three trocars. The patients were discharged upon favorable outcome after surgery. No recurrence of ileus was noted post-surgery. Laparoscopy was useful both to diagnose and treat ileus of a Meckel's diverticulum.
\end{abstract}

\section{Keywords}

Meckel's Diverticulum, Ileus, Preoperative Diagnosis, Laparoscopic Surgery

\section{Introduction}

In recent years, remarkable advances have been made in laparoscopic surgery. Its applications have been gradually increasing, and a number of studies have demonstrated the effectiveness of laparoscopic surgery to diagnose

"Corresponding author.

How to cite this paper: Naritomi, K., Tanaka, Y., Ureshino, H., Uchino, Y., Kuwahara, M., Kitazato, Y., Kawabata, M. and Mano, M. (2016) Three Inverted Meckel's Diverticula Cases with lleus Diagnosed Preoperatively and Treated Laparoscopically. Surgical Science, 7, 65-71. http://dx.doi.org/10.4236/ss.2016.72009 
a Meckel's diverticulum, which is widely recognized as difficult before surgery. Here, we present the case histories of three patients who undergo laparoscopic surgery and are diagnosed with ileus due to an inverted Meckel's diverticulum before surgery and discuss this in the context of the current literature.

\section{Case Presentations}

\subsection{Case 1}

Patient: The patient was a 26-year-old man.

Chief complaint: The chief complaint was upper left abdominal pain.

History: The patient had no history of laparotomy. He experienced ileus and underwent conservative therapy at the age of 24 years.

Medical conditions: The patient experienced upper left abdominal pain after dinner.

On the same day, he sought help at the emergency outpatient service of our hospital.

Status presents at the time of visit to our hospital: He had a blood pressure of 140/75 mmHg, a pulse rate of 80 beats/min and a body temperature of $37.2^{\circ} \mathrm{C}$.

Abdominal findings: The patient felt mild tenderness in the upper left abdomen. The region was flat and soft. No abdominal muscle guarding sign.

Blood biochemical findings: Blood test findings were as follows: white blood cell (WBC) count: 10,200/ $\mu$ l [percentage of neutrophils (NE): $84.2 \%$ ]; C-reactive protein (CRP) level: $0.2 \mathrm{mg} / \mathrm{dL}$. No other abnormal findings were noted.

Findings from abdominal X-ray: Several expanded images of the gas around the small intestine were observed (Figure 1(a)).

Abdominal contrast-enhanced CT: The region 50- to $70-\mathrm{cm}$ from the terminal ileum on the adoral side revealed a diverticulum-like structure. The region contained fecaloid air content. Expansion of the intestine was identified on the adoral side (Figure 1(b)).

After hospitalization: Ileus due to an inverted Meckel's diverticulum was tentatively diagnosed from the above findings, a long tube was inserted on the same day, and decompression therapy was initiated. The abdominal symptoms gradually started to improve. However, it was unclear why the ileus occurred. To clarify this enigma we performed an oral small bowel series. The results demonstrated a saccular structure similar to a Meckel's diverticulum seen on CT (Figure 1(c)). In addition, Meckel's diverticulum scintigraphy was performed, and no sign of abnormal accumulation, such as ectopic gastric mucosa, was observed. Consequently, surgery was performed 12 days from the onset of symptoms due to repeated diagnoses of ileus caused by a Meckel's diverticulum.

Surgical findings: laparoscopy was performed from the three trocars. A $15 \mathrm{~mm}$ vertical incision was made immediately under the umbilicus using the Hasson's approach. The each trocar was placed in the lower right abdomen $(5 \mathrm{~mm})$ and upper right abdomen $(5 \mathrm{~mm})$. Ascites were not detected. A Meckel's diverticulum was observed in the adoral side of ileum, $70 \mathrm{~cm}$ from the ileocecal area. Mild adhesions between the mesentery of the small intestine and Meckel's diverticulum were excised after ablation using GIA (60 mm) staplers in the diverticular roots (Figure 1(d)).

\subsection{Case 2}

Patient: The patient was a 29-year-old man.

Chief complaint: The chief complaint was lower abdominal pain.

History: The patient had no history of laparotomy. At 21 and 28 years of age he experienced ileus that were treated with a conservative therapy.

Medical conditions: One morning, the patient gradually started to experience lower abdominal pain. On the same day, he visited our hospital.

Status presents at the time of the visit to our hospital: He had a blood pressure of 120/64 mmHg, a pulse rate of 80 bears $/ \mathrm{min}$, and a temperature of $37.5^{\circ} \mathrm{C}$.

Abdominal findings: He had mild tenderness in the lower abdomen and no muscle guarding.

Blood biochemical findings: His blood test findings were as follows: WBC count: 13,900/ $\mu 1$ (NE: 81\%); CRP: $0.2 \mathrm{mg} / \mathrm{dL}$. No other abnormal findings were noted. 


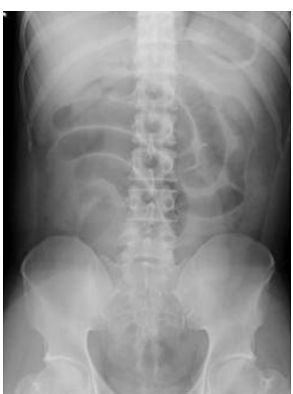

(a)

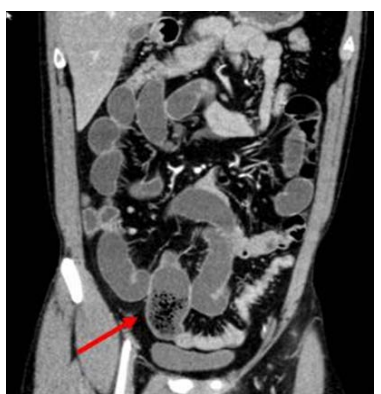

(b)

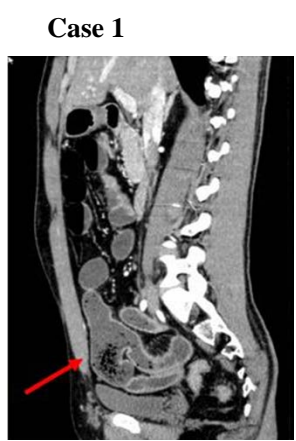

(c)

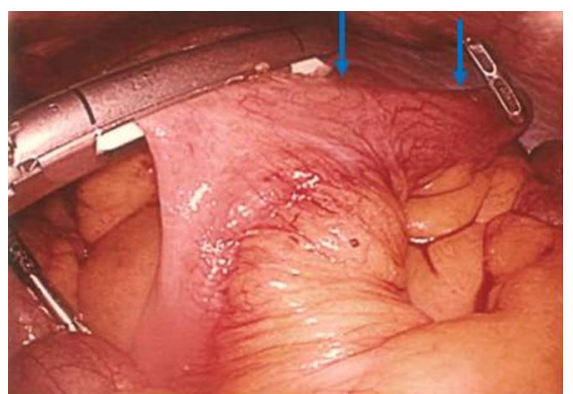

(d)

Figure 1. Case 1. (a) Findings from a simple photo of the abdomen: Several expanded images of the gas around small intestine were detected; (b) and (c) Abdominal contrast-enhanced CT: The region 50- to 70-cm from the terminal ileum on the adoral side revealed a diverticulum-like structure. The region contained fecaloid air. Intestinal expansion was identified on the adoral side; (d) A Meckel's diverticulum was observed.

Findings from abdominal X-ray: Images of the gas expanded small intestine were observed (Figure 2(a)).

Abdominal contrast-enhanced CT findings: Expansion was seen in the lumen of the small intestine. A large volume of fluid has accumulated and sparse niveau formation was observed.

On the left side of right internal iliac artery, a bag-like structure $74 \times 37 \mathrm{~mm}$ in size was caudally protruding from the ileum. Fecal content was found in this region (Figure 2(b)).

After admission: Ileus due to an inverted Meckel's diverticulum was tentatively diagnosed from the above findings and a long tube was inserted on the same day to begin decompression therapy. Although the abdominal symptoms gradually improved, it remained unclear why the ileus kept recurring. To clarify the cause we conducted a long tube contrast. The results showed a saccular structure similar to a Meckel's diverticulum on CT. (Figure 2(c)). Meckel's diverticulum scintigraphy was performed, with no clear sign of abnormal accumulation such as ectopic gastric mucosa. Consequently, surgery was performed 12 days from the onset of symptoms due to repeated diagnoses of ileus caused by a Meckel's diverticulum.

Surgical findings: We performed laparoscopy from the three trocars placed in the lower abdomen $(12 \mathrm{~mm})$, right lower quadrant $(5 \mathrm{~mm})$, and right-upper quadrant $(5 \mathrm{~mm})$. Intraperitoneal observation identified a $10 \mathrm{~cm}$ diverticulum in the ileum as well as mild adhesion between the mesentery and the diverticulum. There was no spread of the inflammation to the surrounding areas. We created a $4 \mathrm{~cm}$ skin incision in the region around the lower abdomen to access the ileum extraperitoneally. Meckel's diverticulum was resected using an 80-mm GIA stapler. We performed interrupted suture of the muscular layer of serosa with 4 - 0 Vicryl thread (Figure 2(d)).

\subsection{Case 3}

Patient: The patient was a 22-year-old man.

Chief complaints: The chief complaints were vomiting and abdominal pain.

History: The patient had no notable history and never had a laparotomy.

Medical conditions: In the evening the patient started to experience vomiting and abdominal pain. On the same day, he visited our hospital.

Status presents: He had a blood pressure of 130/75 $\mathrm{mmHg}$, a pulse rate of 86 beats/min, and a temperature of $37.3^{\circ} \mathrm{C}$.

Abdominal findings: Flatulence was present in the entire abdomen region. Marked tenderness was seen in the umbilical region. No muscle guarding sign.

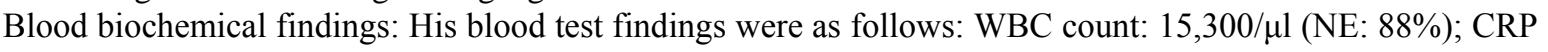
level: $0.7 \mathrm{mg} / \mathrm{dL}$. No other abnormal findings were noted.

Findings from abdominal X-ray: Expanded gas images of the small intestine were detected (Figure 3(a)).

Abdominal contrast-enhanced CT: The source of intestinal stenosis was found on the right ventral side of the right common iliac artery. Fecal content was identified on the adoral side. A sac-like intestine was protruding from the left ventral side of the ileum toward the anus indicating a Meckel's diverticulum. The same region was involved at the end of the ileum causing ileus (Figure 3(b)). 


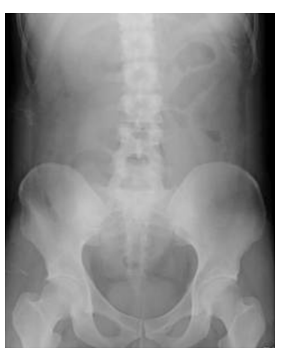

(a)

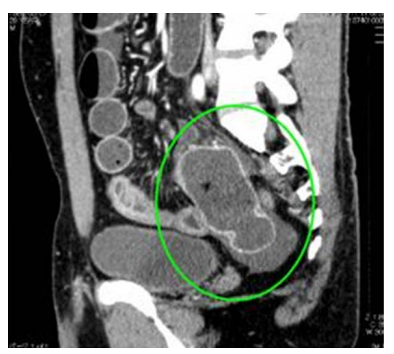

(b)
Case 2

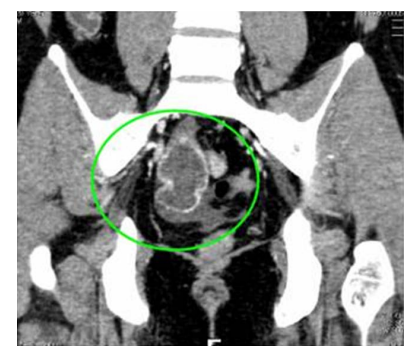

(c)

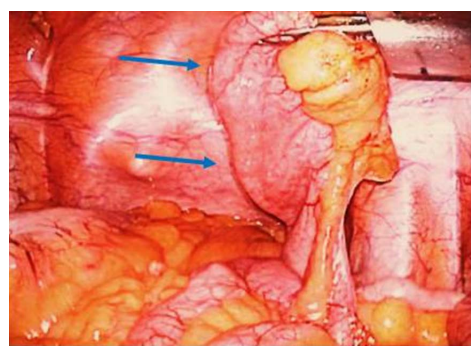

(d)

Figure 2. Case 2. (a) Abdominal X-ray: Enhanced intestinal gas is shown; (b) and (c) Abdominal contrast-enhanced CT findings: On the left side of right internal iliac artery, a bag-like structure $74 \times 37 \mathrm{~mm}$ in size protruded caudally from the ileum. Fecal content was found around in this region; (d) Intraperitoneal observation identified a $10 \mathrm{~cm}$ diverticulum in the ileum as well as mild adhesion between ligament and the diverticulum.

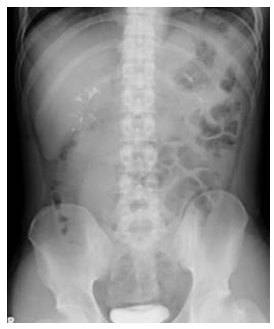

(a)

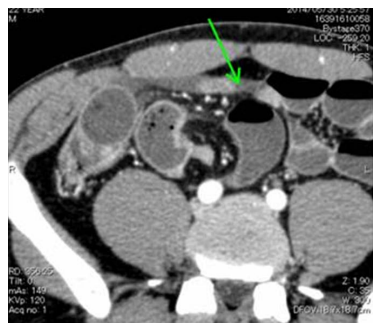

(b)

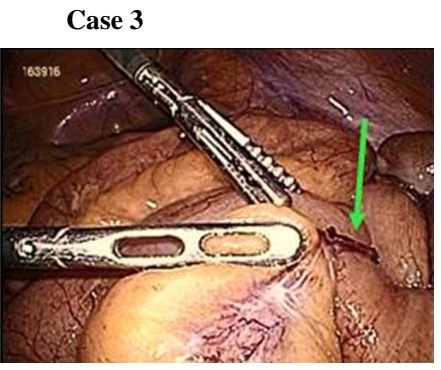

(c)

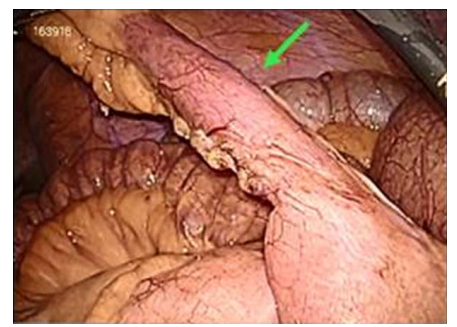

(d)

Figure 3. Case 3. (a) Abdominal X-ray: Enhanced intestinal gas is shown; (b) A sac-like intestine was protruding from the left ventral side of the ileum toward the anus indicating a Meckel's diverticulum. The same region was involved at the end of the ileum causing ileus; (c) A Meckel diverticulum of 7 to $8 \mathrm{~cm}$ was detected in the ileum, $70 \mathrm{~cm}$ from the adoral side of Bauhin's valve. Inflammation was found on top and from that area funicular objects stretched toward the mesentery. Funicular objects were mildly strangulating the ileum; (d) Strangulation was easily reserved after resecting the funicular objects.

After hospitalization: Ileus due to an inverted Meckel's diverticulum was tentatively diagnosed from the above findings, and the patient underwent emergency surgery on the same day.

Surgical findings: Using laparoscopy, a $1.5 \mathrm{~cm}$ trocar was inserted through a small incision above the umbilicus. An expanded small intestine and a moderate amount of pale ascites were identified. A $15 \mathrm{~mm}$ trocar was inserted in left abdominal region. A $5 \mathrm{~mm}$ trocar was placed in the right abdominal region and the suprapubic region. A Meckel diverticulum of 7 to $8 \mathrm{~cm}$ in length was detected in the ileum, $70 \mathrm{~cm}$ from the adoral side of Bauhin's valve. Inflammation was seen on top of that area and funicular objects stretched toward the mesentery. Funicular objects were mildly strangulating the ileum. Strangulation was easily reversed after resecting the funicular objects. The roots of the Meckel diverticulum were resected with a 60-mm GIA stapler (Figure 3(c) and Figure 3(d)). A comparison of the three cases is shown in Table 1. The three cases were not recurrented in our outpatient clinic.

\section{Discussion}

According to a survey of 580 adults with Meckel's diverticulum, the rate of complications are highest among those with ileus (58.7\%), followed by diverticulitis (4.3\%), bleeding, (11.3\%), and perforation (7.5\% [1]). Several previous reports have attempted to elucidate the mechanism underlying ileus due to an inverted Meckel's diverticulum. According to Rutherford et al. [2], ileus is caused by a Meckel's diverticulum due to the following five factors: 1) abnormal diverticulum torsion; 2) strangulation due to Littre hernia; 3) intussusceptions; 4) mesodiverticular band (persistence of the vitelline vessel nourished on yolk duct); and 5) adhesions due to diverticulitis. Two of the cases presented here were caused by adhesions while another was attributed to funicular objects.

Therefore, it is advisable to consider an inverted Meckel's diverticulum when conducting a differential diagnosis of idiopathic ileus for adults without history of laparotomy. 
Table 1. A comparison of the three inverted Meckel's diverticula cases.

\begin{tabular}{|c|c|c|c|}
\hline & Case 1 & Case 2 & Case 3 \\
\hline Age (y.o.) & 26 & 29 & 22 \\
\hline Sex & M & M & M \\
\hline History of Laparotomy & - & - & - \\
\hline Decompression & + & + & - \\
\hline $\begin{array}{l}\text { Meckel's diverticulum scintigraphy } \\
\text { (Accumulation) }\end{array}$ & $\stackrel{+}{(-)}$ & $\stackrel{+}{(-)}$ & - \\
\hline Pathogenesis Operation (day) & 12 & 12 & 1 \\
\hline Developmental mechanism & Adhesion & Adhesion & Strangulation \\
\hline Resection & diverticulum & diverticulum & diverticulum \\
\hline Operation time (min) & 84 & 59 & 72 \\
\hline Discharge (day) & 9 & 13 & 5 \\
\hline
\end{tabular}

However, preoperative diagnosis of Meckel's diverticulum is often difficult.

To establish the presence of diverticulum, we could have performed an oral small bowel series, small intestine endoscopy, or Meckel's diverticulum scintigraphy. Regrettably, it is not appropriate to perform these examinations on patients with an acute abdomen who require urgent care. Furukori [3] and Hidaka et al. [4] reported that this diagnostic method involves visualizing an intestinal structure that continues toward the small intestine and ends at the cecum on CT. In recent years, multi-detector-row CT (MDCT) has allowed the visualization of lesions that were difficult to observe previously. A diagnosis of Meckel's diverticulum was suspected with MDCT in three of our cases at the time of admission. In two of the cases, a long tube was inserted into the bowel to improve the ileus, and a surgery was performed after Meckel's diverticulum was identified using examinations such as a small bowel series. However, the third case received emergency surgery on the same day based on the results of MDCT alone. Thus, it is clear that MDCT was very useful in this diagnosis.

In addition, recent advances in laparoscopic surgery have been increased its applications every year. Many reports have demonstrated the effectiveness of laparoscopic surgery for ileus [5]-[7]. Many recent reports outside of Japan have used diagnostic laparoscopy for unexplained bleeding and abdominal pain; a number of cases involved resecting Meckel's diverticulum laparoscopically to reach a diagnosis. Furthermore, even when the small intestine was only partially resected due to severe adhesion or inflammation in the surrounding area, a small incision made by extending the wounded area around the trocars enables intestinal anastomosis to be safely performed under direct vision with no notable problems [8]-[12]. Takemura et al. [13] argued that a laparoscopic surgery can be safely adapted to treat adhesive intestinal obstruction by using a long tube to facilitate intestinal decompression before surgery and specifying the source of the problem by diagnostic imaging. Sufficient preoperative decompression of the intestine allowed identification of the problem and enabled surgery in our two cases.

The ileus case that was most suitable for endoscopic surgery involves cases of simple adhesive intestinal obstruction with minimal improvement despite using preoperative decompression with a long tube. On the other hand, cases that are not suitable for endoscopic surgery include those with markedly unstable respiratory physiology and obvious strangulating intestinal obstruction, adhesive intestinal obstruction cases that are prominently not receptive to preoperative decompression, and those involving colorectal cancer.

The three basic methods for resecting Meckel's diverticulum are as follows: 1) resection at the base of the diverticulum along the lines of the ileal wall (simple diverticulectomy); 2) wedge resection of the ileum with diverticulum (wedge resection); 3) partial resection of the ileum with diverticulum and an end-to-end anastomosis (partial resection of the ileum). Method 1) has the risk of leaving ectopic mucosa that could cause bleeding while method; 2) could cause ileal stenosis if wedge resection were too deep. Sanders et al. [9] introduced the following surgical guidelines for a Meckel's diverticulum with complications: a) Resecting the base of diverticulum is sufficient for a simple case of ileus caused by a mesodiverticular band; b) Cases of perforated or in- 
flamed diverticulum require resection of abnormal structure. Thus, wedge resection of both the diverticulum and the adjacent ileum will be necessary; c) Cases with bleeding require sleeve resection (segmental resection) including ileum adjacent to diverticulum. In addition, verifying the presence of ulcers in the resected specimen is crucial.

Mukai et al. [14] focuses on the length of Meckel's diverticulum and its location relative to ectopic mucosa. They recommend simple diverticulectomy in cases with a long Meckel's diverticulum, and partial resection of the ileum in cases with a short diverticulum. Our cases involved resection only at the base of diverticulum. In two of these cases we believe this was sufficient for the following reasons: 1) no abnormal accumulation was detected by Meckel's diverticulum scintigraphy prior to surgery and; 2) histopathological findings later confirmed the absence of ectopic gastric mucosa. If the onset of the symptoms is triggered by bleeding, it may be possible to leave ectopic mucosa. In such cases, a partial resection should be considered. Next, we considered the placement position of the first trocar. In laparoscopic surgery, the first trocar is commonly inserted through a small incision around the umbilicus. In laparoscopic surgery, the first trocar is commonly inserted through a small incision around the umbilicus.

However, Chiba et al. [15] advises against insertion around the umbilicus because a Meckel's diverticulum is one morphology of umbilicus disease. The risk that funicular objects are present in the omphaloenteric duct cannot be eliminated. Furthermore, Mike et al. [16] suggest that placing the first trocar in the lower left abdomen makes observation of the entire small intestine easier due to the anatomical location of the mesentery root. In fact, it is impossible to identify the pathogenesis of ileus even when ileus is due to an inverted Meckel's diverticulum. Although we attempted laparoscopy by inserting the first trocar from the umbilical region in the presented cases, it is reasonable to consider other locations for the first trocar in the future. Laparoscopic surgery is remarkably effective in patients who have no surgical history or severe ileus. Differential diagnosis of a Meckel's diverticulum should be conducted using MDCT and other strategies to enhance diagnostic and therapeutic outcomes.

\section{Conclusion}

We reported three cases that were diagnosed as ileus due to an inverted Meckel's diverticulum prior to surgery and underwent a laparoscopic surgery. Each of our cases was diagnosed as ileus, but none had a history of laparotomy. In two cases, we performed laparoscopic surgery after decompression with a long tube. In another case, laparoscopic surgery was conducted on the day of admission due to limited expansion of the intestinal tract. If expansion of the intestine was moderate, it was possible to perform laparoscopic surgery for ileus due to an inverted Meckel's diverticulum.

\section{Acknowledgements}

We thank the Editor in submitting this report.

\section{Competing Interests}

The authors declare that they have no competing interests.

\section{Authors' Contributions}

KN made substantial contributions to the concept and design, and is the main author. YT, HU, and MK performed the surgeries in these cases together. MK reviewed this article and provided advice. MM is our chief supervisor who received permission before manuscript submission.

\section{References}

[1] Yamaguchi, T., Takeuchi, S., Murakuni, H., Awatsu, S., Hoshino, M. and Shinomiya, N. (1976) A Case of Meckel’s Diverticulum Diagnosed with 99 m Tc-Pertechnetate Scanning of the Abdomen and a Review of 580 Cases from Japanese Literature. Journal of Japan Surgical Association, 31, 1647-1651.

[2] Rutherford, R.B. and Akers, D.R. (1966) Meckel's Diverticulum; A Review of 148 Pediatric Patients, with Special Reference to the Pattern of Bleeding and to Mesodiverticular Vascular Bands. Surgery, 59, 618-626. 
[3] Furukori, M., Hasegawa, K., Ohara, K., et al. (2013) A Case of Perforation of Meckel's Diverticulum Diagnosed Preoperatively and Managed by Laparoscopic Treatment Surgery. Journal of Abdominal Emergency Medicine, 33, 883-886.

[4] Hidaka, A., Morinaga, A., Kuroda, H., et al. (2010) Two Cases of Meckel's Diverticulum Diagnosed with the Preoperative Use of Contrast-Enhanced Computed Tomography(CT) of the Abdomen. Journal of Japan Surgical Association, 71, 1190-1194.

[5] Ohta, T., Kano, N., Kusanagi, H., et al. (2013) A Case of Intestinal Obstruction Caused by Meckel’s Diverticulum Successfully Treated by Laparoscopic Surgery. Journal of Japanese College of Surgeons, 38, 129-134.

[6] Yoshizawa, Y., Sanada, H., Ikeda, T. and Kumada, K. (1994) Laparoscopic Reduction for Volvulus Caused by Fibrous Cord Remnant of the Vitelline Duct. Pediatric Surgery, 26, 58-63.

[7] Sanada, H., Nemoto, H., Chiba, M., Yoshizawa, Y., Midorikawa, T., Ikeda, T., et al. (1997) Laparoscopic Procedure for Meckel's Diverticulum and Other Vitelline Duct Anomalies. JSES, 2, 331-335.

[8] Teitelbaum, D.H., Polley Jr., T.Z. and Obeid, F. (1994) Laparoscopic Diagnosis and Excision of Meckel's Diverticulum. Journal of Pediatric Surgery, 29, 495-497. http://dx.doi.org/10.1016/0022-3468(94)90075-2

[9] Sanders, L.E. (1995) Laparoscopy in the Management of Meckel’s Diverticulum. Surgical Endoscopy, 9, $724-727$. http://dx.doi.org/10.1007/BF00187950

[10] Chan, K.W., Lee, K.H. and Mou, J.W.C. (2008) Laparoscopic Management of Complicated Meckel's Diverticulum in Children: A 10-Year Review. Surgical Endoscopy, 22, 1509-1512. http://dx.doi.org/10.1007/s00464-008-9832-0

[11] Craigie, R.J., Forrest, N. and Nanthakumaran, S. (2006) Laparoscopy in Diagnosis and Management of Meckel’s Diverticulum. Journal of Laparoendoscopic \& Advanced Surgical Techniques and Part A, 17, 70-73. http://dx.doi.org/10.1089/lap.2006.16.70

[12] Rivas, H., Cacchione, R.N. and Allen, J.W. (2003) Laparoscopic Management of Meckel’s Diverticulum in Adults. Surgical Endoscopy, 17, 620-622. http://dx.doi.org/10.1007/s00464-002-8613-4

[13] Takemura, M., Ikebe, T., Mayumi, K. and Hamano, G. (2012) Laparoscopic Management of Small Bowel Obstruction; Review of 11 Cases. Journal of Japanese College of Surgeons, 37, 912-916.

[14] Mukai, M., Takamatsu, H. and Noguchi, H. (2002) Does the External Appearance of a Meckel's Diverticulum Assist in Choice of the Laparoscopic Procedure? Pediatric Surgery International, 18, 231-233. http://dx.doi.org/10.1007/s003830100663

[15] Chiba, M., Sanada, H. and Ikeda, T. (1999) Laparoscopic Approach to Meckel's Diverticulum. The Japanese Journal of Gastroenterological Surgery, 22, 864-869.

[16] Mike, M., Kimura, K. and Kiyosawa, Y. (1998) Assisted Laparoscopic Resection for Intestinal Obstruction. Operation, 52, 711-715.

\section{List of abbreviations Used}

Multi-detector-row CT: MDCT 